\title{
Entanglement View of Dynamical Quantum Phase Transitions
}

\author{
Stefano De Nicola®, Alexios A. Michailidis $\odot$, and Maksym Serbyn $\odot$ \\ IST Austria, Am Campus 1, 3400 Klosterneuburg, Austria
}

(Received 27 August 2020; accepted 23 December 2020; published 29 January 2021)

\begin{abstract}
The analogy between an equilibrium partition function and the return probability in many-body unitary dynamics has led to the concept of dynamical quantum phase transition (DQPT). DQPTs are defined by nonanalyticities in the return amplitude and are present in many models. In some cases, DQPTs can be related to equilibrium concepts, such as order parameters, yet their universal description is an open question. In this Letter, we provide first steps toward a classification of DQPTs by using a matrix product state description of unitary dynamics in the thermodynamic limit. This allows us to distinguish the two limiting cases of "precession" and "entanglement" DQPTs, which are illustrated using an analytical description in the quantum Ising model. While precession DQPTs are characterized by a large entanglement gap and are semiclassical in their nature, entanglement DQPTs occur near avoided crossings in the entanglement spectrum and can be distinguished by a complex pattern of nonlocal correlations. We demonstrate the existence of precession and entanglement DQPTs beyond Ising models, discuss observables that can distinguish them, and relate their interplay to complex DQPT phenomenology.
\end{abstract}

DOI: 10.1103/PhysRevLett.126.040602

Introduction.-The rapid development of different quantum simulation platforms [1,2] fuels the exploration of new nonequilibrium phenomena that can be probed in isolated interacting quantum systems. Because of experimental limitations, phenomena observable at short times in quantum quenches are of particular interest. Dynamical quantum phase transitions (DQPTs) have recently emerged as an interesting phenomenon within this regime [3,4]. Since the early work of Heyl et al. [3], who introduced the notion of DQPT considering the quantum Ising model, DQPTs have attracted great interest [5-28]. Moreover, they were experimentally observed in trapped ion quantum simulators [29], superconducting qubits [30], and other platforms [31-34].

In the framework of DQPTs, one considers quantum quenches from an initial state $\left|\psi_{0}\right\rangle$ and monitors the normalized logarithm of the return probability in the process of unitary evolution under a Hamiltonian $H$,

$$
f(t)=-\lim _{L \rightarrow \infty} \frac{1}{L} \log \left|\left\langle\psi_{0}\left|e^{-i H t}\right| \psi_{0}\right\rangle\right|^{2},
$$

where we restrict to one-dimensional cases and denote system size as $L$. This quantity is identified as the nonequilibrium analog of the free-energy density, with DQPTs corresponding to nonanalyticities in the behavior of $f(t)$ at

Published by the American Physical Society under the terms of the Creative Commons Attribution 4.0 International license. Further distribution of this work must maintain attribution to the author(s) and the published article's title, journal citation, and DOI. early times [3]. However, $f(t)$ corresponds to the free energy at complex temperature, and a precise relation between the behavior of $f(t)$ and the equilibrium phase diagram was not established [28]. Phenomenologically, quenches from a state $\left|\psi_{0}\right\rangle$ that realizes a different phase compared to the ground state of $H$ often give rise to DQPTs $[3-5,10,18]$; however, there are exceptions to this rule $[6,7,14,15,27,28]$.

In order to connect DQPTs to equilibrium concepts, such as order parameters, the behavior of local observables was explored [22,26,35]. A direct correspondence was established for systems with broken symmetries, involving a generalized notion of DQPTs [11,17,20,36]. Recently, local string observables capable of revealing DQPTs were introduced [37,38]. However, the general relation between DQPTs and local expectation values remains elusive. Connections to the entanglement entropy were also explored: DQPTs may correspond to regions of rapid growth [29] or peaks [21] in the entanglement entropy, and, for certain quenches in integrable models, they occur at crossings in the entanglement spectrum [9,10,39]. Nonetheless, the underlying mechanism and the conditions under which DQPTs may be related to entanglement signatures are not clearly understood. Thus, in spite of many advancements, the rich phenomenology of DQPTs and their relation to other physical quantities still call for a more general understanding [28].

In this Letter, we utilize the matrix product state (MPS) [40] language for DQPTs that was applied in numerical studies $[5,10,11,14,18,26]$. We show that, in the low-entanglement regime, it is possible to distinguish between precession and entanglement DQPTs, which 

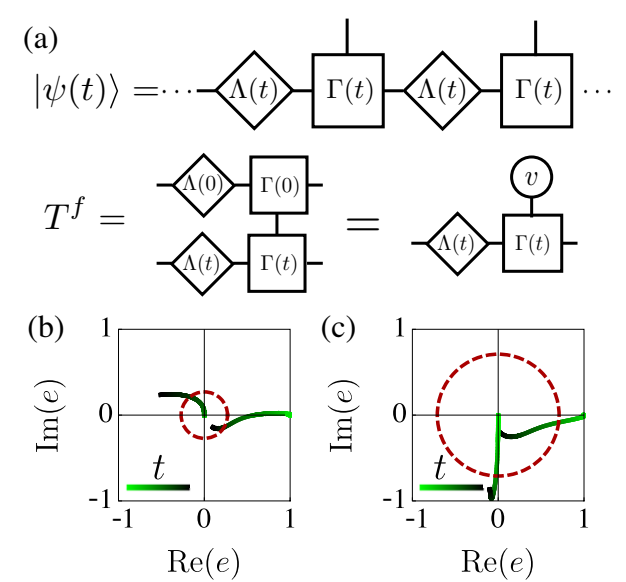
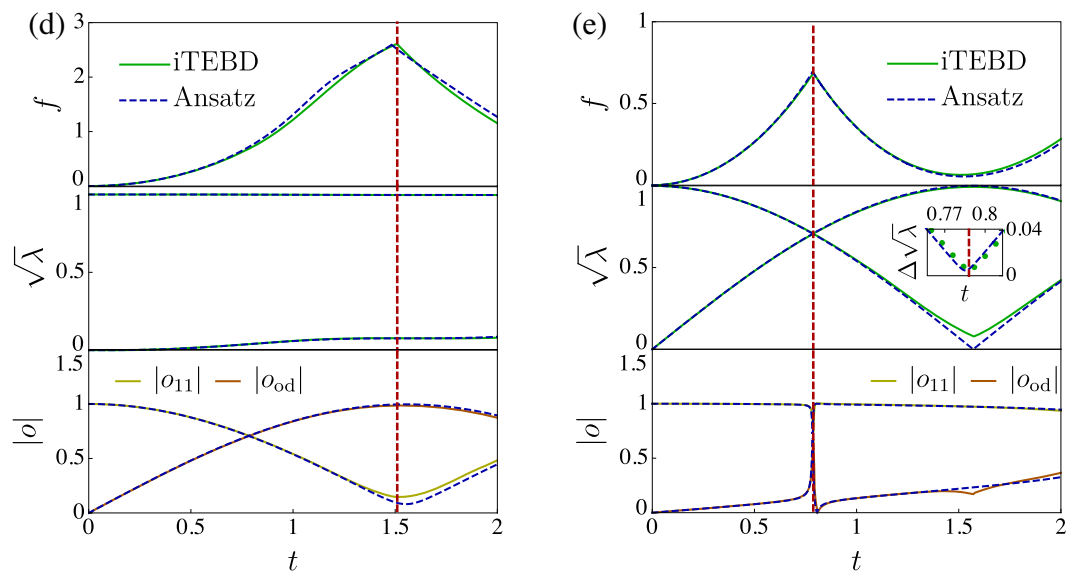

FIG. 1. (a) Representation of the fidelity density transfer matrix $T^{f}$ obtained from the iMPS canonical form when the initial state is a product state. The evolution of the two leading eigenvalues of $T^{f}$ in the complex plane, fidelity density, entanglement spectrum, and the overlaps are illustrated for (b),(d) a pDQPT and (c),(e) an eDQPT. Red circles in (b) and (c) correspond to times where DQPTs occur. (d), (e) Comparison of fidelity density, entanglement spectrum, and overlaps. Solid lines show iTEBD data obtained with $\chi \leq 8$ (truncating $\sqrt{\lambda}_{i}<10^{-9}$ ), and dashed lines correspond to the analytical Ansätze; quench parameters are listed in the main text.

correspond to different physics, as highlighted by analytical MPS Ansätze. We illustrate the existence of entanglement and precession DQPTs in different models, discuss ways to distinguish them experimentally, and suggest how their interplay may lead to the rich phenomenology reported in the literature.

MPS description of DQPTs.-DQPTs are typically studied at short times for quenches from area-law entangled initial states. In this regime, the time-evolved state $|\psi(t)\rangle=$ $e^{-i H t}\left|\psi_{0}\right\rangle$ has area-law entanglement due to Lieb-Robinson bounds [41,42] and admits a MPS description [40]. For translation-invariant initial states (possibly with a finitesize unit cell), infinite MPS (iMPS) [43] provide an efficient representation of $|\psi(t)\rangle$. In Fig. 1(a) we show an iMPS in the canonical form $[43,44]$, where the standard building block of MPS, the tensor $A_{i j}^{\sigma}(t)$, is decomposed as $A_{i j}^{\sigma}(t)=\Lambda_{i i}(t) \Gamma_{i j}^{\sigma}(t)$. Here $\sigma=\uparrow, \downarrow$ is the physical and $i, j=1, \ldots, \chi$ are bond indices. The diagonal matrix $\Lambda_{i i}(t)=\sqrt{\lambda}_{i}$ contains the ordered, $\lambda_{i}>\lambda_{i-1}$, singular values of the Schmidt decomposition across a bond. $\lambda_{i}$ determine the entanglement spectrum, so that the bipartite entanglement entropy is $S=-\sum_{i} \lambda_{i} \log \lambda_{i}$. The tensor $\Gamma^{\sigma}(t)$ carries a physical index and together with $\Lambda$ satisfies a set of canonical conditions, $\sum_{i j \sigma} \Lambda_{i j}^{2} \Gamma_{j k}^{\sigma} \Gamma_{i l}^{\sigma *}=$ $\sum_{i j \sigma} \Lambda_{i j}^{2} \Gamma_{k j}^{\sigma} \Gamma_{l i}^{\sigma *}=\delta_{k l}[44]$.

Using the iMPS representation, the fidelity density is expressed directly in the thermodynamic limit via the spectrum of the fidelity transfer matrix, $T^{f}(t),\left\{e_{i}\right\}$, as [7,45]

$$
f(t)=-2 \log \max \left(\left\{\left|e_{i}\right|\right\}\right) .
$$

The transfer matrix $T^{f}(t)$ is defined in Fig. 1(a) as a contraction of the time-evolved MPS tensor with its conjugate at $t=0$. Thus, $T^{f}(0)$ coincides with the conventional transfer matrix and has $\left|e_{1}\right|=1$ and all other $\left|e_{i}\right|<1$, as follows from normalization of $\left|\psi_{0}\right\rangle$. At later times, the eigenvalues of $T^{f}(t)$ perform complicated evolution in the complex plane. As illustrated in Figs. 1(b) and 1(c), singularities in $f(t)$ emerge from the initially subleading eigenvalue $e_{2}$ surpassing in magnitude the largest one.

Two limiting cases of DQPTs.-To distinguish between different physical mechanisms that drive the crossing between transfer matrix eigenvalues, we use the canonical form of the MPS tensor and focus on the case when the initial state $\left|\psi_{0}\right\rangle=\bigotimes_{i}|v\rangle_{i}$ is a product state. The contraction of the time-evolved MPS with the product state does not affect bond indices [see Fig. 1(a)], resulting in

$$
\begin{aligned}
T^{f}(t) & =\left(\begin{array}{cc}
\sqrt{\lambda_{1}} & 0 \\
0 & \sqrt{\lambda_{2}}
\end{array}\right)\left(\begin{array}{ll}
o_{11} & o_{12} \\
o_{21} & o_{22}
\end{array}\right), \\
o_{i j} & =\sum_{\sigma}\left(v^{\sigma}\right)^{*} \Gamma_{i j}^{\sigma},
\end{aligned}
$$

where we retained the leading $2 \times 2$ part of the MPS virtual space, corresponding to the two largest singular values. For initial product states, the elements of the overlap matrix $o$ are obtained via contraction of the tensor $\Gamma_{i j}^{\sigma}$ with the single-site spinor wave function $v^{\sigma}$.

Equations (2) and (3) single out the contribution of the entanglement spectrum, encoded in the diagonal of the matrix $\Lambda$, to the transfer matrix $T^{f}(t)$ and DQPTs. When the entanglement spectrum features a large gap, $\lambda_{1} \gg \lambda_{2}$, the switch in magnitude between eigenvalues of $T^{f}$ is necessarily driven by the evolution of the overlap matrix. This is a precession DQPT (pDQPT) that is of semiclassical 
nature, as we explain below. In the opposite limit, when the two leading singular values $\lambda_{1}$ and $\lambda_{2}$ exhibit an avoided crossing, the system features entanglement of order $\ln 2$. DQPTs happening near such points are dubbed entanglement DQPTs (eDQPTs). We illustrate these two limits of DQPTs in the quantum Ising model using analytical MPS Ansätze.

Precession DQPTs in the Ising model.-In order to illustrate pDQPTs, we study the dynamics under the transverse- and longitudinal-field Ising model

$$
H=\sum_{i}\left[J \sigma_{i}^{z} \sigma_{i+1}^{z}+h_{x} \sigma_{i}^{x}+h_{z} \sigma_{i}^{z}\right]
$$

The initial state $\left|\psi_{0}\right\rangle=\bigotimes_{i}|\downarrow\rangle_{i}$ is the ground state of the Hamiltonian (4) in the ferromagnetic phase, $J \rightarrow-\infty$, $h_{z}>0$. The evolution is performed with $J=0.1$, $h_{x}=1, h_{z}=0.15$, so that single-spin terms are dominant.

The top panel of Fig. 1(d) shows the fidelity density calculated using infinite time-evolving block decimation (iTEBD) [43]. It exhibits a cusp at $t \approx 1.5$, signaling a DQPT. By bringing the MPS to the canonical form we extract the entanglement and overlap contributions. The middle plot shows the evolution of the two leading singular values, which remain very well separated at the time when the DQPT occurs. At the same time, $\left|o_{11}\right|$ exhibits a minimum near the DQPT, while the off-diagonal component $\left|o_{12}\right|=\left|o_{21}\right| \equiv\left|o_{\text {od }}\right|$ shows a clear maximum. Thus, the overlap matrix is predominantly responsible for the switch of the transfer matrix eigenvalues, providing a prototypical example of pDQPT.

Analytical $p D Q P T$ Ansatz.-The precession nature of pDQPTs can be illustrated by analytically constructing a suitable $\chi=2$ MPS Ansatz. In the limit when $J \ll h_{x}, h_{z}$ we split the Hamiltonian (4) into an interacting part $V=$ $J \sum_{i} \sigma_{i}^{z} \sigma_{i+1}^{z}$ and a free-precessing part $H_{0}=\sum_{i}\left[h_{x} \sigma_{i}^{x}+\right.$ $\left.h_{z} \sigma_{i}^{z}\right]$ that contains only single-spin terms. Then, we move to the rotating frame with respect to $H_{0}$, rewriting the time evolution as $|\psi(t)\rangle=e^{-i H_{0} t} T e^{-i \int_{0}^{t} \tilde{V}\left(t^{\prime}\right) d t^{\prime}}\left|\psi_{0}\right\rangle$. The interaction term in the rotating frame reads $\tilde{V}(t)=e^{i H_{0} t} V e^{-i H_{0} t}=$ $\sum_{i} \sum_{\alpha, \beta} s_{\alpha}(t) s_{\beta}(t) \sigma_{i}^{\alpha} \sigma_{i+1}^{\beta}$, where $\alpha, \beta \in\{x, y, z\}$, the timedependent coefficients are $s_{x}(t)=2 h_{x} h_{z} \sin ^{2}(h t) / h^{2}$, $s_{y}(t)=h_{x} \sin (2 h t) / h$, and $s_{z}(t)=\left[h_{x}^{2} \cos (2 h t)+h_{z}^{2}\right] / h^{2}$, and $h=\sqrt{h_{x}^{2}+h_{z}^{2}}$ is the magnitude of the applied field. Finally, we exploit the slow initial buildup of entanglement along the $z$ axis to replace the $\sigma^{x}$ and $\sigma^{z}$ operators in $\tilde{V}(t)$ by their expectation values under free precession, $-s_{x}$ and $-s_{z}$, respectively. This allows us to approximately write $\tilde{V}(t)$ as a matrix product operator (MPO) of $\chi=2$ (see Supplemental Material [46]). Acting by this MPO on the initial $|\downarrow\rangle$-product state gives a MPS Ansatz for $|\psi(t)\rangle$. Bringing this Ansatz to canonical form [46], we obtain the singular values as $\sqrt{\lambda}_{1}=$ $|\cos [J a(t)]|$ and $\sqrt{\lambda}_{2}=|\sin [J a(t)]|$, where $a(t)=h_{x}^{2}[4 h t-$ $\sin (4 h t)] / 8 h^{3}$. The middle panel of Fig. 1(d) reveals an excellent agreement between our analytical results and iTEBD predictions for the singular values. The $\Gamma$ matrix in the canonical form reads

$$
\Gamma(t)=e^{-i t\left(h_{x} \sigma^{x}+h_{z} \sigma^{z}\right)} e^{-i J b(t) \sigma^{y}}\left(\begin{array}{cc}
|\downarrow\rangle & |\uparrow\rangle \\
i|\uparrow\rangle & -i|\downarrow\rangle
\end{array}\right) \bar{\Lambda},
$$

where $\bar{\Lambda}=\operatorname{diag}(\operatorname{sign}\{\cos [J a(t)]\}, \operatorname{sign}\{\sin [J a(t)]\})$ and $b(t)=h_{x}\left[h_{x}^{2} \cos (6 h t)+3\left(h^{2}+3 h_{z}^{2}\right) \cos (2 h t)-4\left(h^{2}+2 h_{z}^{2}\right)\right] /$ $12 h^{4}$ [46]. The matrix of overlaps $o$ is obtained by contracting all entries of $\Gamma(t)$ with the $\langle\downarrow|$ state on the left. The behavior of $o_{11}$ and $o_{\text {od }}$ obtained from (5) agrees with numerically exact iTEBD results, Fig. 1(d). Since $\lambda_{1} \gg \lambda_{2}$ within the range of considered times, the precession of spins in $\Gamma(t)$ induced by exponentials of Pauli matrices plays the main role in driving the $\mathrm{pDQPT}$.

The dominant component of the MPS corresponds to the top diagonal entry in Eq. (5), and it coincides with the initial state $|\downarrow\rangle$ at $t=0$. The off-diagonal entries in Eq. (5) give subleading contributions suppressed by powers of $\sqrt{\lambda_{2} / \lambda_{1}}$, as follows from Eq. (3). However, as both the dominant component $|\downarrow\rangle$ and its correction $|\uparrow\rangle$ precess, see Eq. (5) and [46], the overlap of the dominant contribution decreases, while the subleading state rotates closer to the $|\downarrow\rangle$ state. A pDQPT occurs when the formerly subleading contribution becomes important enough to flip the magnitude of the eigenvalues of $T^{f}$, which happens when $\left|o_{11} / o_{\text {od }}\right| \sim \sqrt{\lambda_{2} / \lambda_{1}} \ll 1$. The DQPT is then closely associated with the minimum of $o_{11}$, with corrections given by off-diagonal terms; see Fig. 1(d). Note that, although free precession dominates the dynamics for the present quench, a minimal $\chi=2$ is required to capture DQPTs due to Eq. (2), reflecting the quantum nature of such phenomena.

Entanglement DQPTs in the Ising model.-We consider a quench from the initial state $\left|\psi_{0}\right\rangle=\bigotimes_{i}|\rightarrow\rangle_{i}$, corresponding to the free paramagnet ground state of (4) for $h_{x} \rightarrow-\infty$. The dynamics is governed by the Ising Hamiltonian with $J=1, h_{x}=0.1$, and $h_{z}=0.15$. Figure $1(\mathrm{e})$ shows that a DQPT happens near an avoided crossing in the entanglement spectrum. The overlaps $\left|o_{11}\right|$ and $\left|o_{\text {od }}\right|$ also display the evolution characteristic of an avoided crossing. This provides an example of eDQPT.

Analytical eDQPT Ansatz.-The smallness of all but the first two singular values for this quench allows us to analytically construct a $\chi=2$ MPS Ansatz describing eDQPTs, which agrees well with numerically exact iTEBD. To this end, we approximate the time-evolution operator by a second-order Trotter decomposition, splitting the Hamiltonian into a singlespin term $H_{0}$ and a two-spin term $V$. The decomposition reads $e^{-i H t} \approx e^{-i H_{0} t / 2} e^{-i V t} e^{-i H_{0} t / 2}$, where $e^{-i V t}$ admits an exact MPO representation with $\chi=2$ (see Supplemental Material [46]). Applying the resulting MPO to the initial state, we obtain the analytical MPS Ansatz. 


$$
A(t)=\left(\begin{array}{cc}
e^{-i J t} c_{\uparrow}(t)|\uparrow(t)\rangle & e^{i J t} c_{\uparrow}(t)|\uparrow(t)\rangle \\
e^{i J t} c_{\downarrow}(t)|\downarrow(t)\rangle & e^{-i J t} c_{\downarrow}(t)|\downarrow(t)\rangle
\end{array}\right),
$$

where $\left.|\uparrow(t)\rangle=\exp \left[-i t\left(h_{x} \sigma^{x}+h_{z} \sigma^{z}\right) / 2\right)\right]|\uparrow\rangle$ and $c_{\uparrow}(t)=$ $\langle\uparrow \mid \rightarrow(t)\rangle$, and likewise for $\downarrow$.

Casting the Ansatz (6) in canonical form yields the tensor $\Gamma$, which generally has a complicated expression but can be simplified in certain limits [46], and the entanglement spectrum $\lambda_{1,2}=[4 \pm \sqrt{f(t)+13}] / 8$, whose avoided crossings are expected to drive the DQPT. Here $f(t)=$ $4 \cos [4 \theta(t)]-\cos [8 \theta(t)]+8 \sin ^{4}[2 \theta(t)] \cos (4 J t)$ is expressed in terms of a time-dependent angle $2 \cos ^{2} \theta(t)=1+[1-$ $\cos (h t)] h_{x} h_{z} / h^{2}$. The special cases when either $h_{x}$ or $h_{z}$ vanishes correspond to classical [12,22] or integrable $[3,9,10,39,49]$ Ising models, discussed in the Supplemental Material [46].

In the generic case with $h_{x}, h_{z} \neq 0$, the top and middle panels of Fig. 1(e) show that the Ansatz (6) accurately captures the dynamics of the rate function, singular values, and overlaps. The avoided crossing of the singular values leads to a much faster growth of entanglement compared to pDQPTs and drives the switch of the transfer matrix eigenvalues: near the DQPT the quantum state undergoes a rearrangement whereby the initially off-diagonal component, which for $\lambda_{2} \ll \lambda_{1}$ provides a correction to the leading top-diagonal component, becomes the dominant contribution. Thus, eDQPTs manifest a change in the leading component of the quantum state and can be revealed by the structure of nonlocal correlations, as we discuss below.

DQPTs in the XXZ model.-To demonstrate the existence of pDQPTs and eDQPTs beyond the Ising chain, we consider quenches from the fixed initial product state $\left|\psi_{0}\right\rangle=\otimes_{i}|\rightarrow\rangle_{i}$. The dynamics is governed by the $X X Z$ model with a field $H=\sum_{i, \alpha}\left[J_{\alpha} \sigma_{i}^{\alpha} \sigma_{i+1}^{\alpha}+h_{\alpha} \sigma_{i}^{\alpha}\right]$, where $J_{x}=J_{y}$ and we set $h_{y}=0$. Figure 2(a) shows dynamics for $J_{x}=J_{y}=0.9, J_{z}=1, h_{x}=0.1$, and $h_{z}=1$, which displays pDQPTs, as it can be seen from the behavior of the entanglement spectrum in the inset. In Fig. 2(b), we consider the same initial state evolved with $J_{x}=J_{y}=$ $0.3, J_{z}=1, h_{x}=0.3$, and $h_{z}=0.1$. In this case, cusps in $f(t)$ are close to avoided crossings in the entanglement spectrum (see inset), suggesting eDQPTs. The behavior of the overlaps shown in the Supplemental Material [46] confirms these expectations.

Experimental signatures.-pDQPTs and eDQPTs have very different physical mechanisms, yet the fidelity density behaves qualitatively similarly, cf. Figs. 1(d) and 1(e) or Figs. 2(a) and 2(b). An immediate distinction between different DQPTs is provided by the bipartite entanglement entropy $S$ : pDQPTs are of semiclassical nature and occur in low-entanglement regions, whereas eDQPTs are triggered by avoided crossings in $\lambda_{i}$ at early times, reflected in rapid entanglement growth.
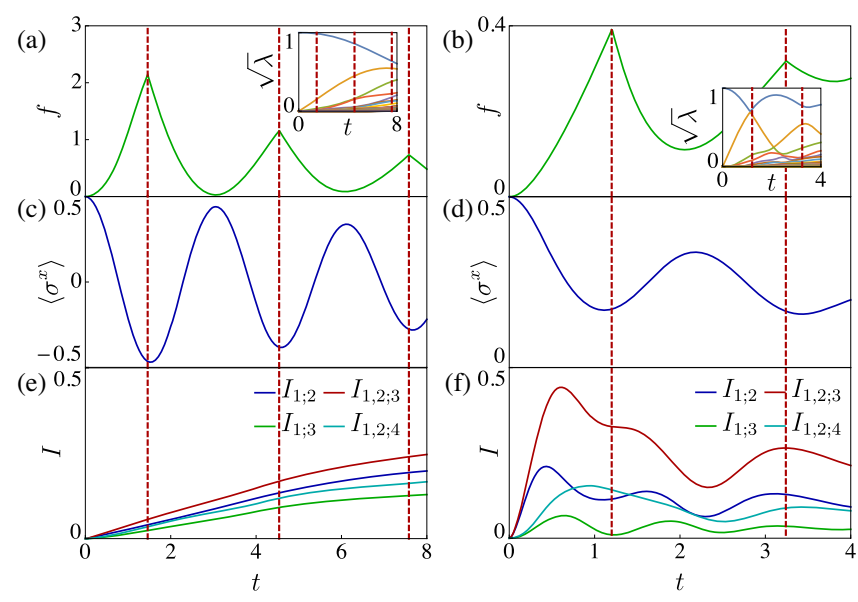

FIG. 2. The qualitative behavior of the fidelity density is very similar for the pDQPTs in (a) and the eDQPTs in (b) that occur in the $X X Z$ spin chain. In contrast, $x$ magnetization and MI have qualitatively different behavior for (c),(e) pDQPTs and (d),(f) eDQPTs. Simulations are performed using iTEBD with $\chi=200$.

While local expectation values evolve smoothly and cannot indicate the precise location of DQPTs, they provide an additional test for the underlying physical mechanisms. Namely, near pDQPTs the dominant component of the state is maximally far away from the initial state, thus the local magnetization along the orientation of the initial state has opposite sign compared to its value at $t=0$, see Fig. 2(c). Near eDQPTs, which are characterized by larger entanglement, local expectation values are expected to be small; this is indeed confirmed by Fig. 2(d), where the magnetization along the $x$ direction assumes its minimal magnitude near an eDQPT.

The mutual information (MI) can be used to reveal the nontrivial entanglement pattern near eDQPTs. The MI between two regions $A$ and $B$ is defined as $I_{A ; B}=$ $S(A)+S(B)-S(A \cup B)$, where $S(\cdot)$ is the von Neumann entropy of a given region. Regions $A$ and $B$ are chosen to contain one or two spins. Because of translational invariance, only relative distances between regions are important. MI provides a basis-independent upper bound on connected correlation functions, which could reveal qualitatively similar behavior provided an appropriate basis is chosen. Figure 2(e) shows that the MI for all choices of regions $A$ and $B$ undergoes slow monotonic growth in the case of a pDQPT. In contrast, eDQPTs correspond to complex oscillatory dynamics of the MI; this is demonstrated in Fig. 2(f), where DQPTs correspond to broad maxima in the MI $I_{1,2 ; 3}$ between spins $\{1,2\}$ and $\{3\}$.

In the Supplemental Material [46], we show a similar pattern for the DQPTs of Figs. 1(d) and 1(e) in the Ising model. The quick growth and nonmonotonic behavior of the MI for eDQPTs signal a change in the dominant component of the wave function. The MI can be probed by the connected correlation functions between the two subsystems, which are typically accessible in experiments. 
Discussion.-We introduced the notions of precession and entanglement DQPTs as two limiting cases, which have different underlying mechanisms and are associated to different physics. pDQPTs can be understood analytically by relying on the large entanglement gap $\lambda_{1} \gg \lambda_{2}$ and the dynamics being driven by single-spin terms in the Hamiltonian. In contrast, eDQPTs happen near avoided level crossings in the entanglement spectrum $\lambda_{1} \sim \lambda_{2} \gg \lambda_{3}$ and can also be analytically described by ignoring $\lambda_{i}$ with $i \geq 3$.

We demonstrated that pDQPTs and eDQPTs exist in different models. These two limits illustrate different physical mechanisms that cause DQPTs, whose relative importance can be qualitatively assessed from the behavior of local observables. Approximations with small bond dimension can then capture DQPTs, provided they incorporate the relevant physics (see Supplemental Material [46]). However, more complicated dynamics emerges when both precession and entanglement production are significant; for instance, in [46] we deform eDQPTs into pDQPTs and show complicated hybrid behavior at intermediate couplings. This suggests that DQPTs are generically the outcome of a combination of factors; the interplay of different mechanisms may then be at the root of the rich phenomenology reported in the literature [4]. Our Letter shows that focusing on the underlying mechanisms is a fruitful path to understanding DQPTs. It would then be interesting to develop analytical Ansätze to characterize situations with more than one dominant mechanism, as well as long-range interacting models $[16,19,20,29]$ and other cases that violate typical phenomenology $[4,19,50]$.

The connection between DQPTs and the spectrum of the fidelity transfer matrix, which is generically non-Hermitian, calls for exploring the relation between DQPTs and the theory of non-Hermitian matrices [51,52], which may allow a classification of DQPTs. Tensor network descriptions could also be used to establish a notion of p-and eDQPTs in higher dimensions and understanding implications for string observables $[37,38]$.

S.D. N. acknowledges funding from the Institute of Science and Technology (IST) Austria and from the European Union's Horizon 2020 Research and Innovation Programme under the Marie SkłodowskaCurie Grant Agreement No. 754411. A. M. and M. S. were supported by the European Research Council (ERC) under the European Union's Horizon 2020 Research and Innovation Programme (Grant Agreement No. 850899).

[1] T. Langen, R. Geiger, and J. Schmiedmayer, Ultracold atoms out of equilibrium, Annu. Rev. Condens. Matter Phys. 6, 201 (2015).

[2] C. Gross and I. Bloch, Quantum simulations with ultracold atoms in optical lattices, Science 357, 995 (2017).
[3] M. Heyl, A. Polkovnikov, and S. Kehrein, Dynamical Quantum Phase Transitions in the Transverse-Field Ising Model, Phys. Rev. Lett. 110, 135704 (2013).

[4] M. Heyl, Dynamical quantum phase transitions: A review, Rep. Prog. Phys. 81, 054001 (2018).

[5] C. Karrasch and D. Schuricht, Dynamical phase transitions after quenches in nonintegrable models, Phys. Rev. B 87, 195104 (2013).

[6] S. Vajna and B. Dóra, Disentangling dynamical phase transitions from equilibrium phase transitions, Phys. Rev. B 89, 161105(R) (2014).

[7] F. Andraschko and J. Sirker, Dynamical quantum phase transitions and the Loschmidt echo: A transfer matrix approach, Phys. Rev. B 89, 125120 (2014).

[8] E. Canovi, P. Werner, and M. Eckstein, First-Order Dynamical Phase Transitions, Phys. Rev. Lett. 113, 265702 (2014).

[9] E. Canovi, E. Ercolessi, P. Naldesi, L. Taddia, and D. Vodola, Dynamics of entanglement entropy and entanglement spectrum crossing a quantum phase transition, Phys. Rev. B 89, 104303 (2014).

[10] G. Torlai, L. Tagliacozzo, and G. D. Chiara, Dynamics of the entanglement spectrum in spin chains, J. Stat. Mech. (2014) P06001.

[11] M. Heyl, Dynamical Quantum Phase Transitions in Systems with Broken-Symmetry Phases, Phys. Rev. Lett. 113, 205701 (2014).

[12] M. Heyl, Scaling and Universality at Dynamical Quantum Phase Transitions, Phys. Rev. Lett. 115, 140602 (2015).

[13] S. Vajna and B. Dóra, Topological classification of dynamical phase transitions, Phys. Rev. B 91, 155127 (2015).

[14] S. Sharma, S. Suzuki, and A. Dutta, Quenches and dynamical phase transitions in a nonintegrable quantum Ising model, Phys. Rev. B 92, 104306 (2015).

[15] M. Schmitt and S. Kehrein, Dynamical quantum phase transitions in the Kitaev honeycomb model, Phys. Rev. B 92, 075114 (2015).

[16] J. C. Halimeh and V. Zauner-Stauber, Dynamical phase diagram of quantum spin chains with long-range interactions, Phys. Rev. B 96, 134427 (2017).

[17] S. A. Weidinger, M. Heyl, A. Silva, and M. Knap, Dynamical quantum phase transitions in systems with continuous symmetry breaking, Phys. Rev. B 96, 134313 (2017).

[18] C. Karrasch and D. Schuricht, Dynamical quantum phase transitions in the quantum Potts chain, Phys. Rev. B 95, 075143 (2017).

[19] I. Homrighausen, N. O. Abeling, V. Zauner-Stauber, and J. C. Halimeh, Anomalous dynamical phase in quantum spin chains with long-range interactions, Phys. Rev. B 96, 104436 (2017).

[20] B. Žunkovič, M. Heyl, M. Knap, and A. Silva, Dynamical Quantum Phase Transitions in Spin Chains with LongRange Interactions: Merging Different Concepts of Nonequilibrium Criticality, Phys. Rev. Lett. 120, 130601 (2018).

[21] M. Schmitt and M. Heyl, Quantum dynamics in transversefield Ising models from classical networks, SciPost Phys. 4, 013 (2018).

[22] D. Trapin and M. Heyl, Constructing effective free energies for dynamical quantum phase transitions in the transversefield Ising chain, Phys. Rev. B 97, 174303 (2018). 
[23] V. Gurarie, Dynamical quantum phase transitions in the random field Ising model, Phys. Rev. A 100, 031601(R) (2019).

[24] S. De Nicola, B. Doyon, and M. J. Bhaseen, Stochastic approach to non-equilibrium quantum spin systems, J. Phys. A 52, 05LT02 (2019).

[25] Y.-P. Huang, D. Banerjee, and M. Heyl, Dynamical Quantum Phase Transitions in U(1) Quantum Link Models, Phys. Rev. Lett. 122, 250401 (2019).

[26] M. Lacki and M. Heyl, Dynamical quantum phase transitions in collapse and revival oscillations of a quenched superfluid, Phys. Rev. B 99, 121107(R) (2019).

[27] R. Jafari, Dynamical quantum phase transition and quasi particle excitation, Sci. Rep. 9, 2871 (2019).

[28] M. Heyl, Dynamical quantum phase transitions: A brief survey, Europhys. Lett. 125, 26001 (2019).

[29] P. Jurcevic, H. Shen, P. Hauke, C. Maier, T. Brydges, C. Hempel, B. P. Lanyon, M. Heyl, R. Blatt, and C. F. Roos, Direct Observation of Dynamical Quantum Phase Transitions in an Interacting Many-Body System, Phys. Rev. Lett. 119, 080501 (2017).

[30] X.-Y. Guo, C. Yang, Y. Zeng, Y. Peng, H.-K. Li, H. Deng, Y.-R. Jin, S. Chen, D. Zheng, and H. Fan, Observation of a Dynamical Quantum Phase Transition by a Superconducting Qubit Simulation, Phys. Rev. Applied 11, 044080 (2019).

[31] N. Fläschner, D. Vogel, M. Tarnowski, B. S. Rem, D.-S. Lühmann, M. Heyl, J. C. Budich, L. Mathey, K. Sengstock, and C. Weitenberg, Observation of dynamical vortices after quenches in a system with topology, Nat. Phys. 14, 265 (2018).

[32] T. Tian, Y. Ke, L. Zhang, S. Lin, Z. Shi, P. Huang, C. Lee, and J. Du, Observation of dynamical phase transitions in a topological nanomechanical system, Phys. Rev. B 100, 024310 (2019).

[33] K. Wang, X. Qiu, L. Xiao, X. Zhan, Z. Bian, W. Yi, and P. Xue, Simulating Dynamic Quantum Phase Transitions in Photonic Quantum Walks, Phys. Rev. Lett. 122, 020501 (2019).

[34] X.-Y. Xu, Q.-Q. Wang, M. Heyl, J. C. Budich, W.-W. Pan, Z. Chen, M. Jan, K. Sun, J.-S. Xu, Y.-J. Han, C.-F. Li, and G.-C. Guo, Measuring a dynamical topological order parameter in quantum walks, Light Sci. Appl. 9, 7 (2020).

[35] C. Rylands and V. Galitski, Dynamical quantum phase transitions and recurrences in the non-equilibrium BCS model, arXiv:2001.10084.

[36] J. Feldmeier, F. Pollmann, and M. Knap, Emergent Glassy Dynamics in a Quantum Dimer Model, Phys. Rev. Lett. 123, 040601 (2019).

[37] J. C. Halimeh, D. Trapin, M. V. Damme, and M. Heyl, Local measures of dynamical quantum phase transitions, arXiv:2010.07307.
[38] S. Bandyopadhyay, A. Polkovnikov, and A. Dutta, Observing dynamical quantum phase transitions through quasilocal string operators, arXiv:2011.03906.

[39] J. Surace, L. Tagliacozzo, and E. Tonni, Operator content of entanglement spectra in the transverse field Ising chain after global quenches, Phys. Rev. B 101, 241107 (R) (2020).

[40] S. Paeckel, T. Köhler, A. Swoboda, S. R. Manmana, U. Schollwöck, and C. Hubig, Time-evolution methods for matrix-product states, Ann. Phys. (N.Y.) 411, 167998 (2019).

[41] E. H. Lieb and D. W. Robinson, The finite group velocity of quantum spin systems, Commun. Math. Phys. 28, 251 (1972).

[42] J. Eisert and T. J. Osborne, General Entanglement Scaling Laws from Time Evolution, Phys. Rev. Lett. 97, 150404 (2006).

[43] G. Vidal, Classical Simulation of Infinite-Size Quantum Lattice Systems in One Spatial Dimension, Phys. Rev. Lett. 98, 070201 (2007).

[44] R. Orús and G. Vidal, Infinite time-evolving block decimation algorithm beyond unitary evolution, Phys. Rev. B 78, 155117 (2008).

[45] L. Piroli, B. Pozsgay, and E. Vernier, Non-analytic behavior of the Loschmidt echo in $X X Z$ spin chains: Exact results, Nucl. Phys. B933, 454 (2018).

[46] See Supplemental Material at http://link.aps.org/ supplemental/10.1103/PhysRevLett.126.040602, which cites Refs. [47] and [48], for details about the analytical MPS Ansätze, further result on DQPTs in the Ising and XXZ models, an illustration of the connection between pDQPTs and eDQPTs, and a discussion of DQPTs in high-entanglement regimes.

[47] G. M. Crosswhite and D. Bacon, Finite automata for caching in matrix product algorithms, Phys. Rev. A 78, 012356 (2008).

[48] G. Mussardo, Statistical Field Theory: An Introduction to Exactly Solved Models in Statistical Physics, Oxford Graduate Texts (Oxford University Press, Oxford, 2009).

[49] P. Calabrese, F. H. L. Essler, and M. Fagotti, Quantum quench in the transverse field Ising chain: I. Time evolution of order parameter correlators, J. Stat. Mech. (2012) P07016.

[50] D. Trapin, J. C. Halimeh, and M. Heyl, Unconventional critical exponents at dynamical quantum phase transitions in a random Ising chain, arXiv:2005.06481.

[51] E. J. Bergholtz, J. C. Budich, and F. K. Kunst, Exceptional topology of non-Hermitian systems, arXiv:1912.10048.

[52] Y. Ashida, Z. Gong, and M. Ueda, Non-Hermitian physics, arXiv:2006.01837. 